\title{
An Overview of DSA via Multi-Channel MAC Protocols
}

\author{
Rodrigo Soulé de Castro, Philippe Godlewski and Philippe Martins \\ Télécom ParisTech, NMS research group \\ France
}

\section{Introduction}

The development of radio access technologies requires an increasing number of spectrum resources. Unfortunately, spectrum bands are scarce and the development of new wireless communication networks and services are thus more and more challenging ([jia07]). Recent reports indicate that fixed channel allocations result in low efficiency in spectrum utilization because a large portion of the spectrum remains underutilized ([mchenry05]).

One approach capable of dealing with the above problem is Dynamic Spectrum Access (DSA) which allows spectrum sharing. In such an approach, unlicensed users, known as secondary users (SUs), dynamically look for unused spectrum in licensed bands and communicate using "spectrum holes". These idle bands represent spectrum portions assigned to licensed users (known as primary users, PUs) that are not being used at a considered time and location ([timmers07]).

Many researchers have proposed different multi-channel MAC protocols to increase network throughput and reduce interference caused by secondary use of the spectrum. Many of these studies consider Wi-Fi like protocols (or IEEE 802.11 based mechanism).

Cognitive Radios (CR) are a type of radio capable of switching channels and adapting its transmission parameters in real-time ([mitola99]). Common MAC protocols do not provide, in general, mechanisms for channel switching. When having multiple independent channels to be used simultaneously, the need for enhanced Multi-channel MAC protocols becomes paramount. The IEEE 802.11 standard uses a distributed coordination function (DCF), as the fundamental Medium Access Control (MAC) technique. However, the distributed coordinate function, which employs carrier sense multiple access with collision avoidance (CSMA/CA), was not designed to work in a multi-channel environment ([ahmed07]).

Secondary users equipped with a cognitive radio, in a multi-channel environment, may improve the efficiency of spectrum utilization and increase the network throughput.

\section{Background}

\subsection{Secondary use of spectrum}

A cognitive radio is an intelligent communication device, which has the ability to adapt its transmission parameters such as channel frequency, modulation and power; based on the interaction with the environment in which it operates ([jia07]). 
There are two different approaches of secondary use of spectrum in cognitive radio context. One is in the form of overlay, opportunistic usage of idle bands in the primary user's (PU) spectrum by cognitive radios and another in the form of underlay, using Ultra Wide Band (UWB) technology ([cabric06]).

The rules in secondary use of frequency spectrum specify that licensed users, known as Primary Users (PUs), have the rights for interference-free communication in certain bands. When these bands are not used by the primary users, they can be used by Secondary Users (SUs). As soon as a primary user starts activity in its channel, the SU has to vacate the channel to avoid interference ([timmers07]). However, a cognitive radio (using a half duplex transceiver) cannot scan the spectrum and transmit simultaneously in the same frequency band. Then, for the protection of primary users, a maximum detection or sensing time must be established. This detection time represents the maximum time of interference, from secondary users, that a primary user can tolerate ([jia07]).

\subsection{Rendezvous in multi-channel protocols}

In multi-channel MAC protocols, Mobile Stations (MSs) exchange control information to concur on the channel for data transmission in the user plane. Proposed protocols vary in how MSs negotiate the channel to be used for data transmission and the way to solve medium contention; these protocols can be divided according to their principle of operation.

In single rendezvous protocols, the rendezvous between a sender and its receiver can take place on at most one channel at any time, while in Multiple Rendezvous protocols, several rendezvous can take place in different channels simultaneously, thereby mitigating the control channel congestion ([mo07]).

In single rendezvous, three different classes of protocols can be distinguished based on the mechanism of channel negotiation ([sheung07]). The Dedicated Control Channel approach, which uses two transceivers (TRx), operates with a single channel only for control packets exchange. In this approach, the MSs always tune one TRx to the control channel to make agreements and be aware of neighbours' negotiations. The other TRx is able to switch channels and is used for data transmission. The Split Phase protocol uses only one TRx for control and data packets. In this protocol, time is split into fixed periods of control and data phases. The control phase is used as common control channel to make rendezvous, when control phase ends, MSs switch to their selected channels and begin data transmission. The third class of protocol is named Common Hopping, which also has only one TRx for both control and data packets, in this protocol there is no CCCH. MSs hop synchronously through all available channels and pauses hopping when sender and receiver agree on data transmission using their current channel.

\subsection{Hidden terminal problem in a single channel environment}

Hidden terminal problem occurs when mobile stations cannot detect signal from other MSs by carrier sensing because they do not have a physical connection to each other. Figure 2 illustrates this problem: MS " $\mathrm{A}$ " sends a message to MS " $\mathrm{B}$ "; " $\mathrm{C}$ " cannot detect the signal from " $A$ " since " $C$ " is out of range of " $A$ ". For station " $C$ ", the channel is idle. When MS " $C$ " sends a message to " $B$ ", this message will collide at " $B$ " with the message sent from " $A$ ". In this scenario " $C$ " is the hidden node to " $A$ ". 


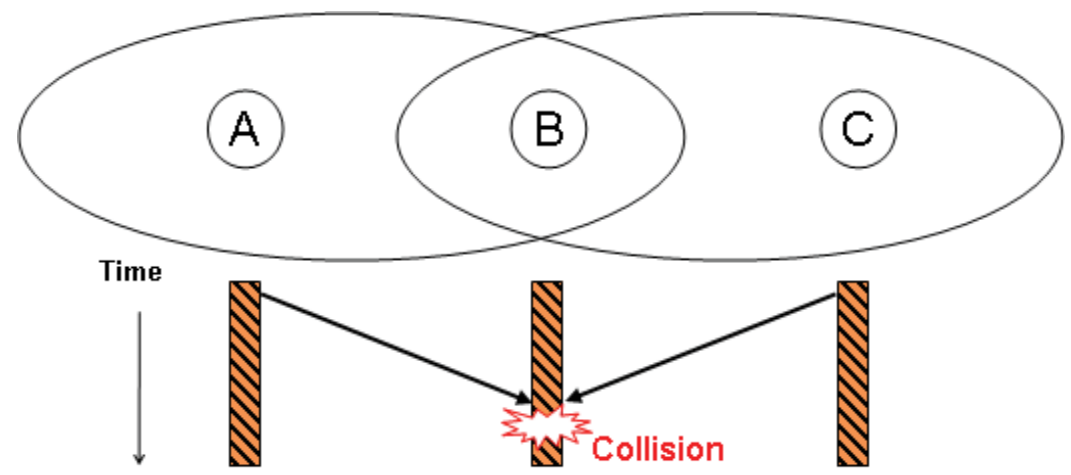

Fig. 2. Hidden terminal problem in a single channel environment

\subsection{Virtual carrier sensing using RTS/CTS exchange}

To deal with the above problem, the IEEE 802.11 MAC layer uses the Distributed Coordination Function (DCF) mechanism, which employs virtual carrier sensing to solve the hidden terminal problem by using the RTS/CTS mechanism.

In this mechanism, when a mobile station wants to initiate communication, it first sends a RTS (Request-To-Send) message and the receiver replies by sending a CTS (Clear-To-Send). The RTS and the CTS contains the NAV (Network Allocation Vector), which is the expected duration of time that other mobile stations, around the communication pair, must refrain from sending data to avoid collisions.

This procedure can solve the hidden terminal problem in a single channel environment, under the assumption that all mobile stations have the same transmission range. However, the DCF mechanism cannot work well in a multi-channel environment, the reason is because MSs may be transmitting or receiving data packets in different channels, missing the RTS/CTS procedure of the DCF mechanism.

\subsection{Multi-channel hidden terminal problem}

This problem occurs when mobile stations in the network listen to different channels missing the RTS/CTS procedure.

The Multi-Channel Hidden Terminal Problem is illustrated in figure 3. Initially, mobile station " $\mathrm{A}$ " wants to communicate with " $\mathrm{B}$ ", then " $\mathrm{A}$ " sends an A-RTS to " $\mathrm{B}$ " on the Common Control Channel (Channel 1). After receiving the A-RTS, MS B selects the Channel 2 to communicate with " $\mathrm{A}$ " and sends back an A-CTS, notifying their neighbours that the data channel number 2 has been selected. In a single channel environment the RTS/CTS exchange avoids collisions in the transmission ranges of " $A$ " and " $B$ ". However, in multichannel environments other mobile stations could be involved in communication in different channels when the RTS/CTS procedure took place. That is the case of mobile stations " $\mathrm{C}$ " and " $\mathrm{D}$ ", as they were communicating in channel 3 they did not hear the A-CTS sent by " $B$ ". When they finish their communication on Channel 3, mobile stations " $C$ " and " $\mathrm{D}$ " switch to Channel 1 and now they select Channel 2 to reinitiate communication. When MS " $C$ " sends the first message to " $\mathrm{D}$ ", this message will cause collision to mobile station " $\mathrm{A}$ " and "B" on Channel 2. 


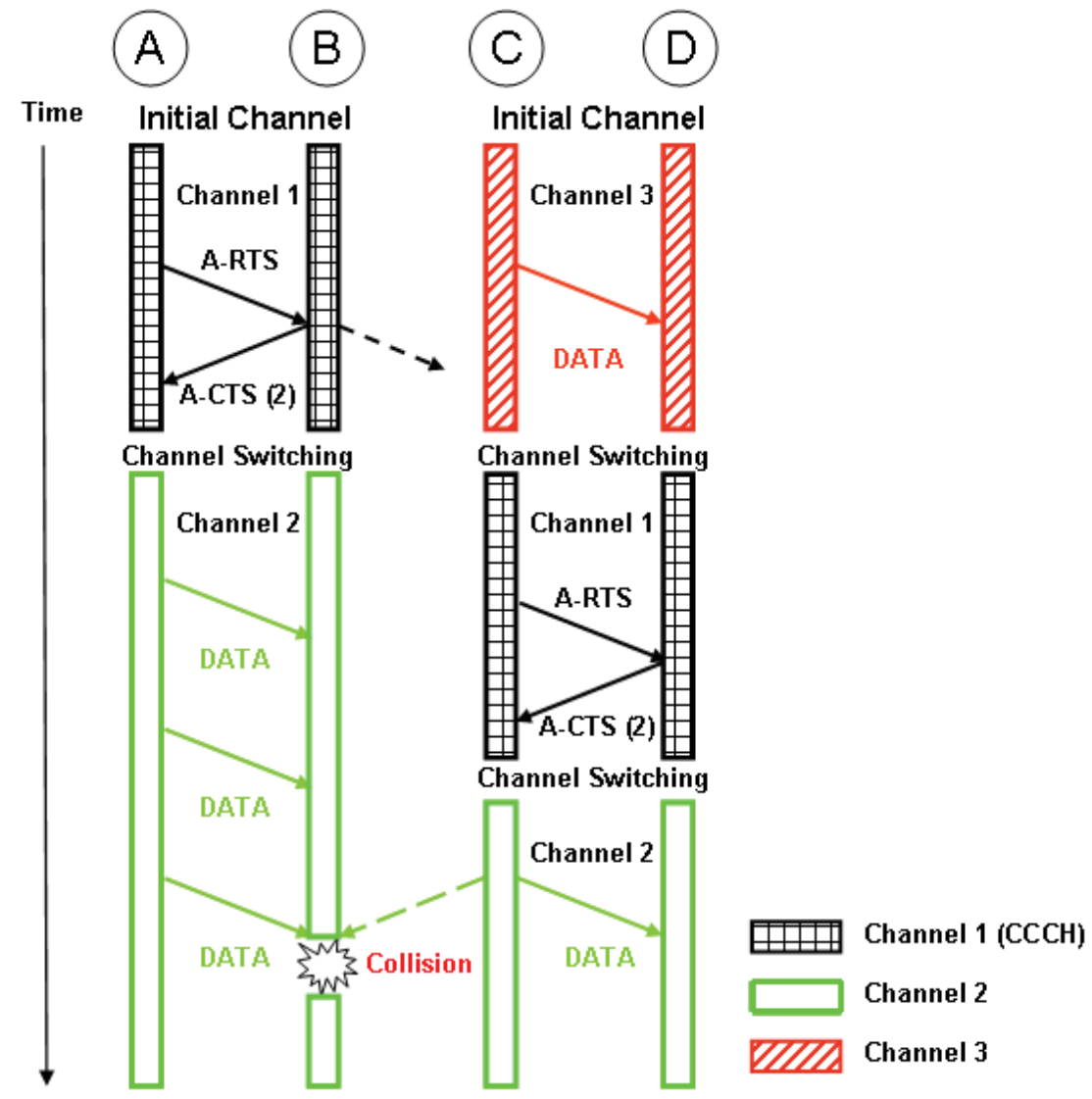

Fig. 3. Hidden terminal problem in Multi-channel protocols (figure inspired from Jungmin So et al. [so04])

One possible solution would be a unique channel or moment in which every MS in the network listens to, thereby, ensuring that the RTS/CTS procedure can be heard by all the MSs, thus avoiding the Multi-Channel Hidden Terminal Problem ([so04]).

\section{Multi-channel MAC protocols}

\section{1 "Comparison of multi channel MAC protocols" [mo07]}

[mo07] presents a performance comparison between different multi-channel MAC protocols, single rendezvous protocols (dedicated control channel, common hopping and split phase) and multi rendezvous (parallel rendezvous).

Dedicated Control Channel Approach: This protocol uses 2 TRx per Mobile Station (MS), one is used for control information exchange and the other is able to switch between channels for data transmission. There is no need for synchronization to make rendezvous because the control channel is always tuned by all the MSs in the network. However, this protocol presents two principal problems, the need for 2 TRx and the possibility of control channel bottleneck. 


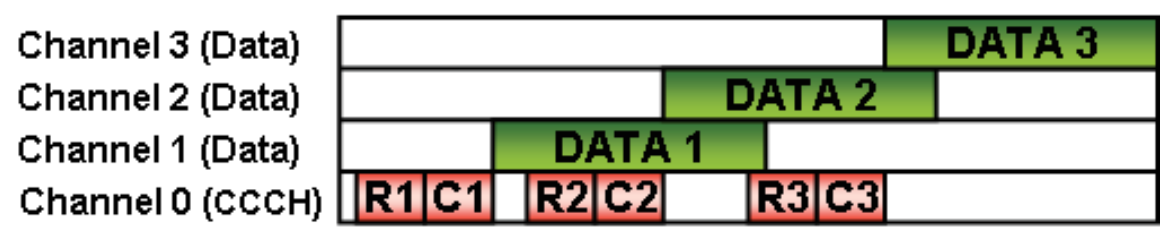

Fig. 4. Dedicated Control Channel Approach (figure inspired from [mo07])

Common Hopping Approach: This protocol uses 1 TRx per Mobile Station (MS); this TRx is able to switch between channels for control information exchange and data transmission. To make rendezvous, MSs hop synchronously over all the channels and pauses its hopping sequence when the agreement between sender and receiver is made. This protocol uses all the channels for data transmission. However, the synchronization among MSs is crucial.

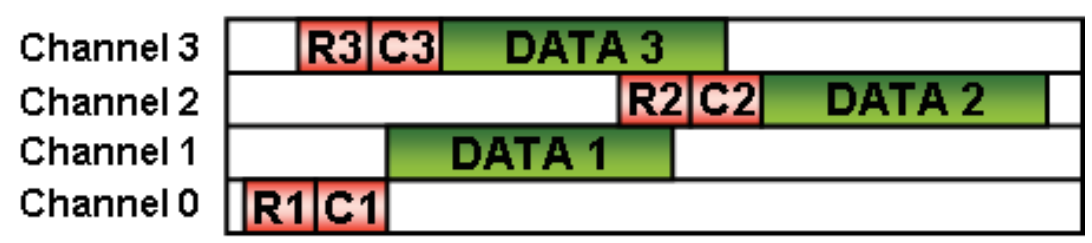

Fig. 5. Common Hopping Approach (figure inspired from [mo07])

Split Phase Approach: This protocol uses 1 TRx per Mobile Station (MS), time is divided into control Phase and Data phase, this division has the objective to ensure that all MSs listen to the control phase, thus avoiding the Multi-Channel Hidden Terminal problem (MCHTP). Two important disadvantages of this protocol are the need for global synchronization and the wasted data channels during the control phase. However, with only one TRx, this protocol solves the MCHTP.

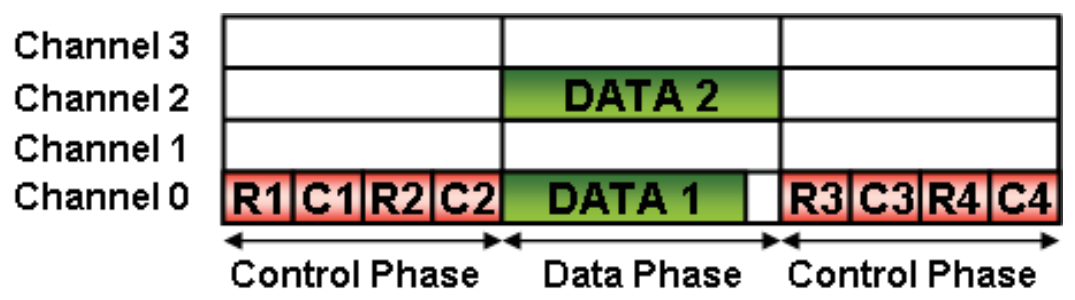

Fig. 6. Split Phase Approach (figure inspired from [mo07])

\section{2 "McMAC: A parallel rendezvous multi-channel MAC protocol” [sheung07]}

McMAC protocol uses 1 TRx per Mobile Station (MS). At the beginning, a sender chooses a hopping pattern in a pseudo-random way using a seed to generate it, neighbours learn its hopping sequence because is included in all the sender's packets. To make rendezvous, a MS can deviate from its default hopping sequence and hops to the receiver's channel. In this protocol multiples rendezvous can be made in different channels at the same time, thus 
improving the network throughput and avoiding control channel bottleneck. However, the synchronization and coordination between MSs are essential.

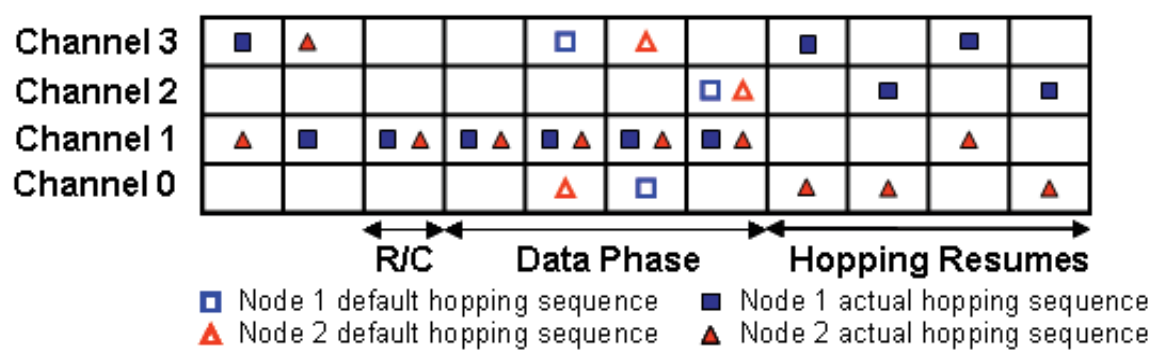

Fig. 7. McMAC protocol (figure inspired from [mo07])

3.3 "SSCH: Slotted Seeded Channel Hopping for capacity improvement in IEEE 802.11 ad-hoc wireless networks" [bahl04]

SSCH protocol uses 1 TRx per Mobile Station (MS). In this protocol, each sender chooses one of the possible hopping patterns generated in a pseudo-random way (one hopping pattern for each available channel). To make rendezvous, a sender must wait until its current hopping pattern intersects with that of the receiver before it can send data. The principal disadvantage of this protocol is the time wasted waiting to coincide with the receiver. However, multiples rendezvous can be made at the same time in different channels and the control channel bottleneck is avoided.

3.4 "Multi-channel MAC for ad hoc networks: handling multi-channel hidden terminals using a single transceiver" [so04]

In MMAC protocol, each MS is equipped with $1 \mathrm{TRx}$. Time is divided into an alternating periods of control and data phases (split phase). An Ad Hoc Traffic Indication Message (AR), at the start of each control interval, is used to indicate traffic and negotiate channels for utilization during the data interval. A similar approach is used in IEEE 802.11's power saving mechanism (PSM). This scheme uses two new packets which are not used in IEEE 802.11 PSM: the ATIM ACK (AC) and the ATIM-RES (A-RE). These packets inform the neighbourhood nodes of the Sender (S) and Destination (D), of which channels are going to be used during the data exchange. During the control period, named ATIM window, all MSs have to attend the default channel and contend for the available channels. Once reservation is successful, the MSs switch to the reserved channel. With only one TRx this protocol solves the Multi-Channel Hidden Terminal Problem. A Preferred Channel List (PCL) is used to select the best channel based on traffic conditions. In this list all the channels are classified by the status: HIGH, MID, and LOW.

The major drawback of the scheme could be the need for synchronizing beacons, which might be difficult to implement in Ad Hoc networks and the waste of the bandwidth in other channels during the ATIM window (control period). However, with only one TRx this protocol solves the MCHTP. 


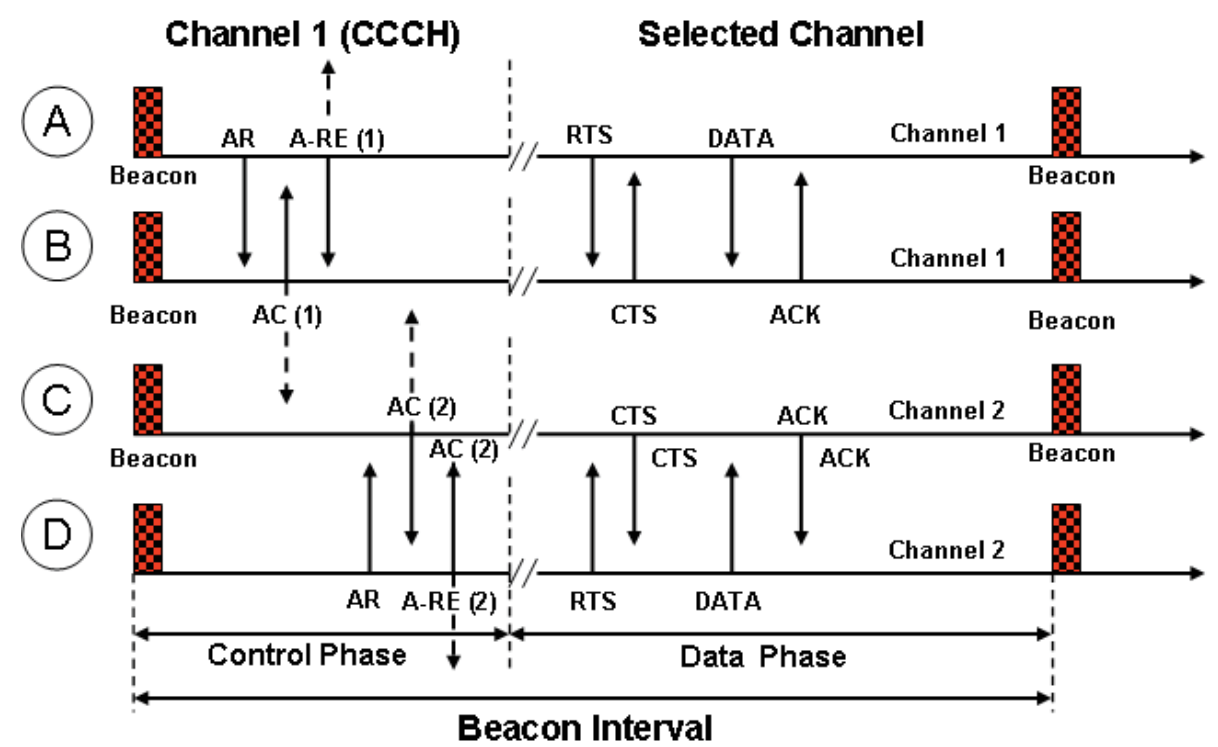

Fig. 8. MMAC protocol (figure inspired from [so04])

\section{5 "A distributed multichannel MAC protocol for cognitive radio networks with primary user recognition" [timmers07]}

In MMAC-CR protocol, time is split into alternating periods of control and data phase and each user is equipped with 1 TRx. A similar approach is used in IEEE 802.11's power saving mechanism (PSM). This protocol has two data structures: the Spectral Image of Primary users (SIP), which contains the channels used by Primary Users (PUs), and the Secondary users Channel Load (SCL), which is used to select the communication channel in terms of traffic.

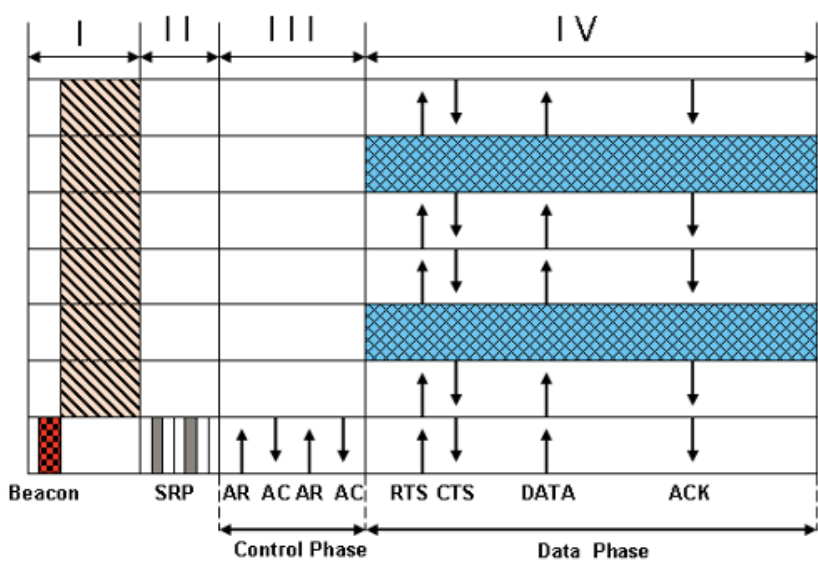

Fast Scan

Busy Slots

Closed Channel

Phases:

I.- Beacon Contention and Fast Scanning

II.- Scan Request

III.- Control Window

IV.- Data Window and

Fine Scanning

Cognitive Control

Channel $(\mathrm{CCCH})$

Fig. 9. MMAC-CR protocol (figure inspired from [timmers07]) 
The proposed protocol is divided into four phases: during phase I, the nodes contend to transmit a beacon and perform a fast scan; this scanning process is used to update the SIP value of the scanned channel. Phase II is used to determine the spectral opportunities by listening to $\mathrm{C}$ minislots (each minislot correspond a data channel). Each MS informs the others of the presence of PUs by transmitting a busy signal in the corresponding minislot. In Phase III, using ATIM packets (AR and AC), the channels are negotiated. Phase IV is used for data transmission or fine sensing for idle nodes.

MMAC-CR with only one TRx solves the "Multi-Channel Hidden Terminal Problem". Alternating periods of control and data phases, this protocol avoids the possibility of control channel bottleneck. However, the synchronization and coordination between MSs are essential to make rendezvous which might be difficult to implement in Ad hoc networks.

\section{6 "TMMAC: an energy efficient multi-channel MAC protocol for ad hoc networks" [zhang07]}

In TMMAC, each user is equipped with $1 \mathrm{TRx}$; time is divided into control phase (ATIM window) and data phase. The ATIM window size is not fixed and can be adapted based on traffic conditions. The data phase is slotted, only a single data packet can be transmitted or received during each time-slot. The purpose of the control window is twofold, the channel negotiation and the slot negotiation. In the data phase, each node switches to the negotiated channel and uses its respective time slot for packet transmission or reception.

This protocol has the same advantages and disadvantages presented in split phase protocols: the need for global synchronization and the wasted data channels during the control phase. However, with only one TRx, this protocol solves the MCHTP.

\section{7 "Hardware-constrained multi-channel cognitive MAC" [jia07]}

In HC-MAC, each MS is equipped with $1 \mathrm{TRx}$. In this protocol, there is no need for global synchronization. To make rendezvous, HC-MAC transfers control packets using a Common Control Channel $(\mathrm{CCCH})$. Time is divided into Contention phase, Sensing phase and Transmission phase and each phase has a RTS/CTS exchange:

1. C-RTS/C-CTS: using the RTS/CST mechanism (cf. IEEE 802.11 DCF mode), a pair of MSs reserves all the channels ( $\mathrm{CCCH}$ and data channels) for the following two phases (sensing and transmission).

2. After sensing the different data channels, the pair exchanges a S-RTS/S-CTS on the $\mathrm{CCCH}$ to mutually inform about channel availability. A set of channels (only one in single Tx case) is then selected.

3. After data transmission on the different selected channels, the communication pair informs the end of transmission by a T-RTS/T-CTS exchange. This allows neighbouring MSs to begin the contention phase with a random back off.

Authors outline two constraints for cognitive radios, sensing and transmission, the former used to optimize the stopping of spectrum sensing and the later used to optimize the spectrum utilized in transmission by secondary users.

The major drawback of this scheme could be that after one communication pair wins the $\mathrm{CCCH}$, using the C-RTS/C-CTS exchange, other mobile stations must defer their sensing and transmission. Then, for a certain time, only one pair uses all available channels and other users must wait for the T-RTS/T-CTS notification to contend again in the control channel. 


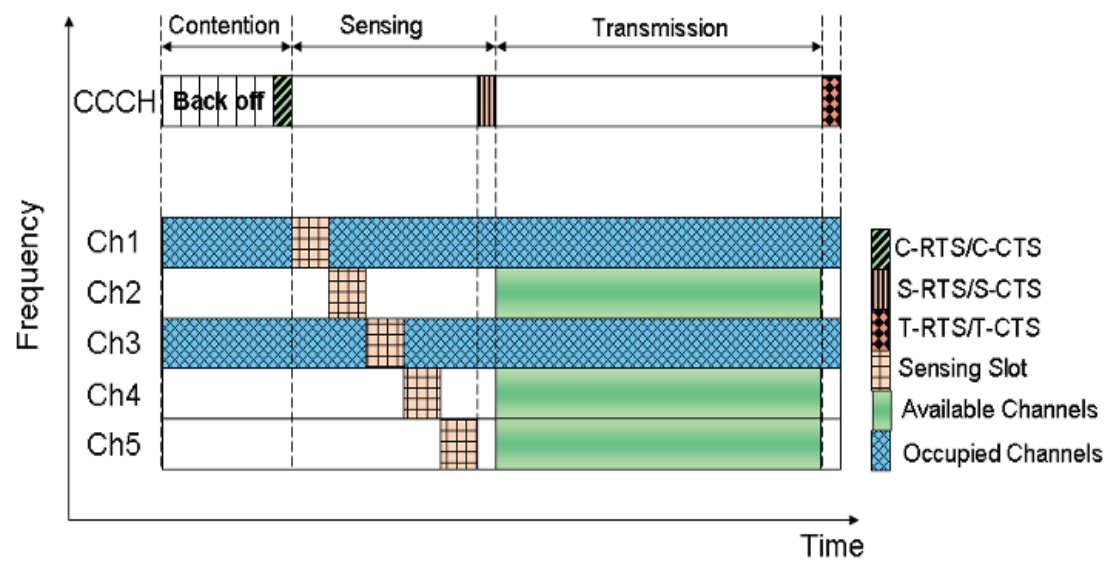

Fig. 10. HC-MAC protocol (figure inspired from [jia07])

\section{8 "Distributed coordinated spectrum sharing MAC protocol for cognitive radio" [nan07]}

This protocol uses 2 TRx per Mobile Station (MS), one is used for control information exchange and the other is able to switch between channels for data transmission. There is no need for synchronization to make rendezvous because the control channel is always tuned by the MSs. In this protocol, secondary users employ a time slot mechanism for cooperative detection of primary users around the communication pair by using the CHRPT (channel report slots). Each node informs the others about the presence of PUs, in the sender and in the receiver side, by transmitting a busy signal in the corresponding minislot (there is one minislot for each data channel).

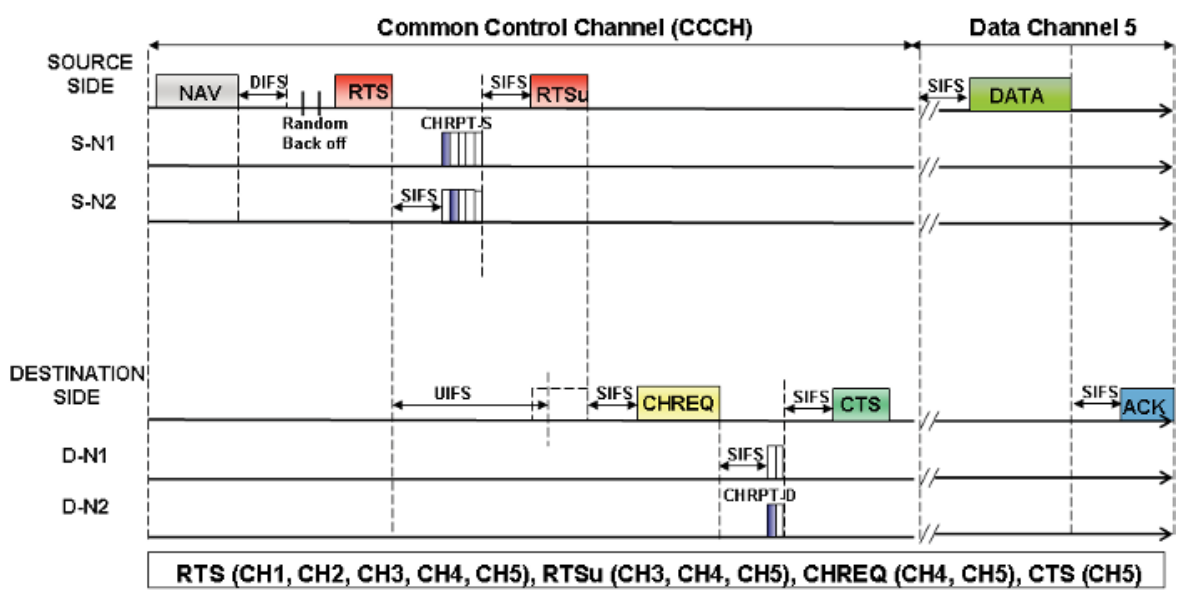

Fig. 11. Procedure of the proposed protocol (figure inspired from [nan07])

The source sends to destination the RTS which includes its available channel list. Neighbour nodes, which hear the RTS, compare the sender list with their own; if they detect a PU 
occupation in a channel, they reply with a pulse in the specified time slot during CHRPT (signalling occupied channels seen by the neighbours). If necessary, the source update its RTS sending a RTSu. The same mechanism occurs in the destination side. After the RTS reception the destination waits to get the possible RTSu for certain time named UIFS, if the RTSu does not arrives, the destination will handle the first RTS. After the RTS reception, the destination sends to its neighbours the Channel Status Request (CHREQ), which includes the destination available channel list among the listed channels of the source. At the end of channel verification by the destination neighbours, the receiver sends the CTS with the chosen channel.

The major drawbacks of the scheme are the time wasted in channel verification by the neighbours and the need for two TRx. However, this procedure ensures the absence of primary users in the vicinity of the communication pair.

\section{9 "Performance of multi channel MAC incorporating opportunistic cooperative diversity" [ahmed07]}

In CD-MMAC, time is divided into fixed periods (split phase), each user is equipped with 1 TRx. This protocol uses the same mechanism proposed by So et al. in MMAC ([so04]). The authors of this protocol add the notion of relays between source and destination. Time is divided into fixed-time intervals (control phase and data phase) using beacons, a small window, named ATIM, at the start of each interval is used to indicate traffic and negotiate channels to be used during the data phase. This protocol uses intermediate nodes as relays to increase the probability of transmission success.

This protocol solves the MCHTP with only one TRx. However, two drawbacks of CDMMAC are the need for global synchronization and the wasted data channels during the control phase.

\subsection{0 "A full duplex multi channel MAC protocol for multi-hop cognitive radio networks" [choi06]}

In this protocol, each secondary user is equipped with 3 TRx named: "Receiver, Transmitter and Controller". To communicate, the RECEIVER of the receiving node and the TRANSMITTER of the sending node must be tuned to the same channel.

There is no need for synchronization because the $\mathrm{CCCH}$ is always tuned by the MSs using the CONTROLLER. A MS selects an unused frequency band as its home channel (HCh), it tunes its receiver to its $\mathrm{HCh}$ and informs the others about its selected channel by broadcast in the control channel. This protocol uses CSMA/CA scheme of IEEE 802.11 DCF mode. With the use of three TRx, MSs can reduce communication delay by transmitting packets while they are receiving. However, the need for 3TRx will increase the overall cost.

\subsection{1 "A multi channel MAC for opportunistic spectrum sharing in cognitive networks" [mishra06]}

In AS-MAC (Ad hoc SEC Medium Access Control) protocol, the primary user is a TDMA/FDMA (GSM) cellular network and the secondary user is an Ad hoc network that can decode the control information of GSM system. Sensing the vacant slots, the SU uses the resources left utilized by the primary user, which could be a Base Station (BS) or a Mobile Station (MS). To obtain all the parameters like synchronization, frequency correction and 
cell information, secondary users decode the beacon channel from the BS. To make rendezvous, this protocol employs RTS/CTS and Reservation (RES) mechanism.

\subsection{2 "Performance evaluation of a medium access control protocol for IEEE 802.11s mesh networks" [benveniste06]}

CCC protocol uses 2 TRx per Mobile Station (MS), one is used for control information exchange and the other is able to switch between channels for data transmission. There is no need for global synchronization to make rendezvous because the control channel is always tuned by the MSs. The CCC protocol defines a Common Control Channel (CCCH), over which, mesh nodes will exchange control and management frames, the rest of the channels, called Mesh Traffic (MT) channels, are used to carry the data traffic. Reservations of the various MT channels are made by exchanging control frames on the $\mathrm{CCCH}$.

This protocol has the same advantages and disadvantages presented by the dedicated control channel approach: there is no need for synchronization to make rendezvous. However, this protocol needs two TRx and the possibility of control channel bottleneck exists.

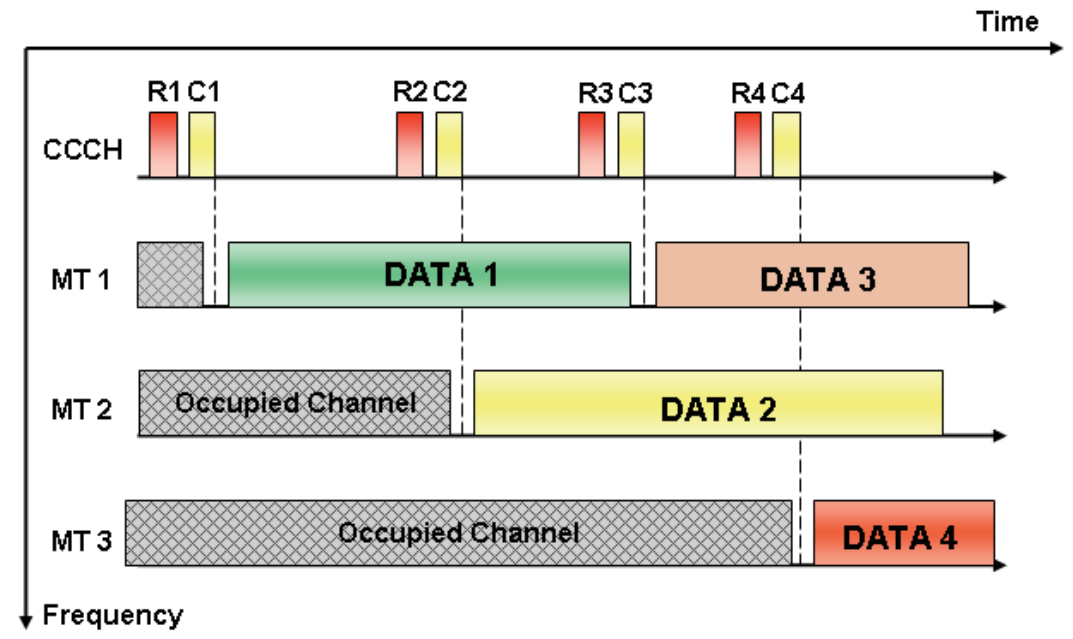

Fig. 12. CCC MAC protocol (figure inspired from [benveniste06])

\section{4. "Os-MAC: an efficient MAC protocol for spectrum-agile wireless networks" [hamdaoui08]}

In Os-MAC protocol, each secondary user is equipped with $1 \mathrm{TRx}$; this protocol uses the IEEE 802.11 DCF mode. This approach seeks to exploit the available spectrum opportunities using MSs coordination. One entity per channel is a "delegate", the delegates are chosen among MSs and makes reports about channel quality. A single ACK notion is used in a "multicast group" named Secondary User Group (SUG).

OS-MAC divides time into periods; each period is named Opportunistic Spectrum Period (OSP). In each OSP, there exist three consecutive phases: Select, Delegate, and Update Phase. In the first phase, each SUG selects the "best" Data Channel (DC) based on traffic conditions 
and uses it for communication during the totality of the OSP period. During the second phase, a Delegate Secondary User (DSU) is chosen to represent the data channel during the Update Phase, in which, all DSUs switch to the CCCH to update each other about their channel conditions, mean while, all non-DSUs continue communicating on their DCs.

An important aspect of this protocol is the notion of groups and the Delegate for each DC. This mechanism can improve the channel classification necessary to define the best channel, based in traffic conditions, which could be used for data transmission.

\section{1 "Primary Channel Assignment Based MAC (PCAM) a multi channel MAC protocol for multi-hop wireless networks" [pathma04]}

In PCAM protocol, each user is equipped with $3 \mathrm{TRx}$. This scheme eliminates the need for a dedicated control channel that arise the possibility of control channel bottleneck when the traffic increases. In this protocol, a MS selects a frequency band as its primary channel, this will be used as a receiver channel and a secondary channel is used as transmitter while the third TRx is used for transmitting and receiving broadcast messages. PCAM protocol removes the constraints of time synchronization and control channel saturation because the channels are pre-assigned. However, the need for 3 TRx will increase the overall cost and the channel assignment procedure, in this protocol, is not specified.

\section{2 "Adaptive MAC protocol for throughput enhancement in cognitive radio networks" [lee08]}

In this protocol, each user is equipped with $2 \mathrm{TRx}$, this protocol proposes two channels, the first one is a WLAN channel which is always available for data transmission; the second one, named "Cognitive channel", is available sporadically. When traffic conditions restrain the use of the cognitive channel, this channel is used for frame errors recovery by transmitting the same information in both channels, known as frequency diversity in MIMO systems; otherwise, the cognitive channel can be used to increase the overall throughput by sending sequential frames using both channels.

The drawback of this scheme could be the need for two TRx. However, this procedure can enhance the overall throughput if the "Cognitive channel" is available.

\section{3 "CREAM-MAC: An efficient Cognitive Radio-EnAbled Multi-channel MAC protocol for wireless networks" [su08]}

In the Cognitive Radio-EnAbled Multi-channel MAC (CREAM-MAC) protocol, each secondary user is equipped with $1 \mathrm{TRx}$ that can dynamically utilize one or multiple channels to communicate and also has multiple sensors that can detect multiple channels activity simultaneously. This protocol needs neither centralized controllers nor synchronization.

The CREAM-MAC protocol employs a Common Control Channel (CCCH) as the "rendezvous channel". With one TRx, this protocol solves the Multi-Channel Hidden Terminal Problem employing a four-way handshake. These control packets are RTS/CTS and CST/CSR, the RTS/CTS exchange prevents the collisions among the secondary users by reserving the $\mathrm{CCCH}$ for channel negotiation. The CST/CSR exchange avoids collisions between secondary and the primary users by allowing secondary users to share sensing information about PU's channel occupation. 


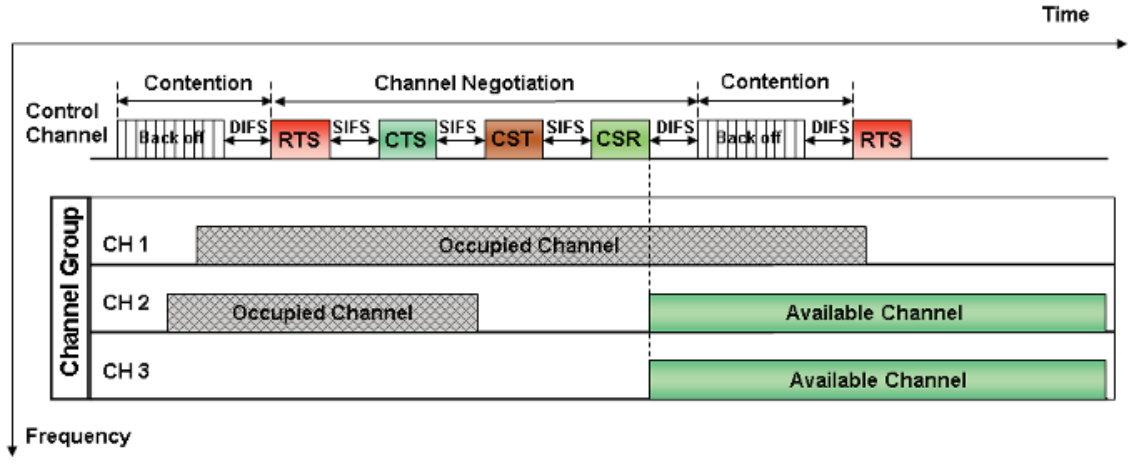

Fig. 13. CREAM-MAC protocol (figure inspired from [su08])

The merit of the CREAM-MAC protocol is the fact that there is no need for global synchronization and with the use of only one TRx and multiple sensors, this protocol solves the MCHTP.

\section{4 "Distributed coordination in dynamic spectrum allocation networks" [zhao05]} In this paper, the notion of groups with similar views of spectrum availability is addressed. Each secondary user is equipped with $1 \mathrm{TRx}$, this protocol employs a voting scheme for selection of a "Coordination Channel" $(\mathrm{CCH})$ for a group and this "user group" is assembled based in similar spectrum channel availabilities.

The $\mathrm{CCH}$ is used as the only means to connect secondary users, thus, only members of the same group can directly communicate with each other. To maintain network connectivity "bridge" nodes, located on the edge of each group, must manage at least two different $\mathrm{CCH}$ to transfer data packets between groups and connect users with different spectrum perspective.

The advantage of this approach is its possible application in the case of secondary use of the spectrum by WLAN devices in TV white spaces, principally, because the interference condition with primary users is determined by distance.

\section{5 "Single-Radio Adaptive Channel Algorithm for spectrum agile wireless ad hoc networks" [ma07]}

In the Single-Radio Adaptive Channel (SRAC) algorithm, each secondary user is equipped with 1 TRx. This algorithm proposes an adaptive channelization, where a radio combines multiple fixed channels with minimum bandwidth, named "atomic channels", based on its needs to form a new channel with more bandwidth, thus forming a "Composite channel". In this algorithm there is no need for global synchronization. SRAC also proposes "Crosschannel communication", utilized to enable communications when there are multiple jamming sources and there is no common idle spectrum between the transmitter and the receiver. A node always has a pre-assigned channel for reception, which is well known by its neighbours and will be used to reach that node; this channel can be modified but the selection must follows strict rules to enable future communications.

The merits of this algorithm are the adaptive channelization and the fact that it does need neither $\mathrm{CCCH}$ nor synchronization because the MSs have a pre-assigned channel for reception. 


\section{6 “Cognitive radio system using IEEE 802.11a over UHF TVWS” [ahuja08]}

This paper presents a practical implementation of IEEE 802.22 WLAN/TV with Primary and Secondary users. The architecture consists of Cognitive Mobile Stations (CMS) and a Cognitive 802.11 Access Point (CAP), which performs band sensing and available channel determination. The Cognitive Access Point has $1 \mathrm{TRx}$ and $1 \mathrm{Rx}$ for sensing, the Cognitive Mobile Stations are equipped with only $1 \mathrm{TRx}$. There is no $\mathrm{CCCH}$, the CAP sends a broadcast message to inform all stations about the available channels list and time synchronization. A Geo-location module is used to guarantees that the cognitive radio units will never transmit on a channel that is determined to be within a licensed station's protected contour.

\section{7 "Spectrum sharing radios" [cabric06]}

This paper proposes the utilization of overlay, opportunistic usage of idle bands, for data transmission and underlay, using UWB technology, for control messages exchange. In this approach, authors propose two different types of control channels. The first one is a low throughput and wide coverage channel, named "Universal Control Channel (UCC)", which is used as a $\mathrm{CCCH}$ allowing the co-existence of several Radio Access Technologies (RATs). The second type of channel, named Group Control Channel (GCC), works as "Group Coordination Channel". This channel with high throughput and short coverage allows sensing information exchange, link maintenance and performs channel allocation.

The advantage of the use of UWB Control Channels is that we could have a realistic and reliable Cognitive Control Channel, always free of Primary users, which is one of the principal assumptions in several propositions of Multi-Channel MAC protocols.

\section{Conclusions}

This chapter presents the main existing multi channel MAC protocols. The merits of several protocols are discussed with regard to different factors: the number of transceivers, the need for synchronization, the need for a common control channel $(\mathrm{CCCH})$ and the different ways to make rendezvous for data transmission. As we showed, each multi-channel MAC protocol faces and resolves differently the various complications that arise in dynamic spectrum access.

In short, Cognitive Radio (CR) technology offers the possibility for additional use of radio spectrum by secondary users. Multiple channel protocols allow dynamic spectrum access (DSA) due to the fact that different rendezvous and data transmissions can be performed on different channels. This type of protocols, compared to others that use a single frequency channel (IEEE 802.11mechanism), may improve spectrum utilization and increase total network throughput.

\section{Acronyms}

ATIM: Ad hoc Traffic Indication Message

AC: ATIM ACK

AR: ATIM

A-CTS: ATIM CTS (which includes the data channel selection)

A-RE: ATIM Reservation

A-RTS: ATIM RTS (which includes the data channel selection) 
$\mathrm{CCCH}$ : Common Control Channel

CR: Cognitive Radio

CREAM-MAC: Cognitive Radio-EnAbled Multi-Channel MAC protocol proposed in [su08]

CSR: Channel-State-Receiver

CST: Channel-State-Transmitter

DC: Data Channel

DCF: Distributed Coordination Function \{IEEE 802.11\}

DSU: Delegate Secondary User

MCHTP: Multi-Channel Hidden Terminal Problem

MC-MAC: Multi-Channel (wireless) MAC

MMAC: Multi-Channel MAC protocol proposed in [so04]

MMAC-CR: Multi-Channel MAC protocol proposed in [timmers07]

MS: Mobile Station

OSMAC: Opportunistic Spectrum Media Access Control proposed in [hamdaoui08]

OSP: Opportunistic Spectrum Period

PN: Primary Network

PSM: Power Saving Mechanism

PCL: Preferred Channel List

PU: Primary User

RAT: Radio Access Technology

SCL: Secondary users Channel Load

SIP: Spectral Image of Primary users

SU: Secondary User

SUG: SU Group

TRx: Transceiver

\section{References}

[ahmed07] Sabbir Ahmed, Christian Ibars, Aitor del Coso and Abbas Mohammed, Performance of Multi Channel MAC incorporating Opportunistic Cooperative Diversity. in IEEE Vehicular Technology Conference, April 2007.

[ahuja08] Ramandeep Ahuja, Robert Corke and Alan Bok, Cognitive Radio System using IEEE 802.11a over UHF TVWS. New Frontiers in Dynamic Spectrum Access Networks, 2008.

[bahl04] Paramvir Bahl, Ranveer Chandra and John Dunagan, SSCH: Slotted Seeded Channel Hopping for Capacity improvement in IEEE 802.11 Ad-Hoc Wireless Networks. in MobiCom 2004.

[benveniste06] Mathilde Benveniste and Zhifeng Tao, Performance Evaluation of a Medium Access Control Protocol for IEEE 802.11s Mesh Networks, in Sarnoff Symposium, 2006.

[cabric06] Danijela Cabric, Ian D. O'Donnell, Mike Shuo-Wei Chen, and Robert W. Brodersen, Spectrum Sharing Radios, in IEEE Circuits and Systems Magazine, 2006.

[choi06] Noun Choi, Maulin Patel and S.Venkatesan, A Full Duplex Multi channel MAC Protocol for Multi Hop Cognitive Radio Networks, in Cognitive Radio Oriented Wireless Networks and Communications Conference, June 2006.

[hamdaoui08] Bechir Hamdaoui and Kang G. Shin, Os-MAC: An efficient MAC Protocol for Spectrum-Agile Wireless Network, in IEEE Transactions on Mobile Computing 2008. 
[jia07] Juncheng Jia and Qian Zhang, Hardware-constrained Multi-Channel Cognitive MAC. in IEEE Global Telecommunications Conference, November 2007.

[lee08] Byungjoo Lee and Seung Hyong Rhee, Adaptive MAC Protocol for Throughput Enhancement in Cognitive Radio Networks, in: Information Networking, 2008.

[mishra06] Amitabh Mishra, A Multi channel MAC for Opportunistic Spectrum Sharing in Cognitive Networks, in Military Communications Conference, 2006.

[mitola99] J. Mitola III, Cognitive radio for flexible mobile multimedia communication, in: Proc. IEEE International Workshop on Mobile Multimedia Communications (MoMuC) 1999, November 1999, pp. 3-10.

[ma07] Liangping Ma, Chien-Chung Shen, and Bo Ryu, Single-Radio Adaptive Channel Algorithm for Spectrum Agile Wireless Ad Hoc Networks, in: New Frontiers in Dynamic Spectrum Access Networks, 2007.

[mchenry05] Mark A; McHenry, NSF, Spectrum Occupancy Measurements, Project Summary, http://www.sharedspectrum.com/ , August 2005

[mo07] Jeonghoon Mo, Hoi-Sheung Wilson So and Jean Walrand, Comparison of Multi channel MAC protocols, in IEEE Transactions on Mobile Computing 2007.

[nan07] Hao Nan, Tae-In Hyon and Sang-Jo Yoo, Distributed Coordinated Spectrum Sharing MAC protocol for cognitive radio, 2007.

[pathma04] Jaya Shankar Pathmasuntharam, Amitabha Das and Anil Kumar Gupta, Primary Channel Assignment Based MAC (PCAM) A Multi Channel MAC Protocol for Multi-Hop Wireless Networks, in Wireless Communications and Networking Conference, 2004.

[sahin07] Mustafa E. Sahin, Sadia Ahmed, and Hüseyin Arslan. The Roles of Ultra Wideband in Cognitive Networks, in IEEE International Conference on UltraWideband, 2007.

[sheung07] Hoi-Sheung Wilson So, Jean Walrand and Jeonghoon Mo, McMAC: A Parallel Rendezvous Multi-Channel MAC protocol. in IEEE Wireless Communications and Networking Conference, March 2007.

[so04] Juming So and Nitin Vaidya, Multi-Channel MAC for Ad Hoc Networks: Handling Multi-Channel Hidden Terminals Using A Single Transceiver, in Proceedings of AMC MobiHoc, May 2004.

[su08] Hang Su and Xi Zhang, CREAM-MAC: An efficient Cognitive Radio- EnAbled MultiChannel MAC Protocol for Wireless Networks. in: International Symposium on a World of Wireless, Mobile and Multimedia Networks, 2008.

[timmers07] Michael Timmers, Antoine Dejonghe, Liesbet Van der Perre and Francky Catthoor, A Distributed Multichannel MAC Protocol for Cognitive Radio Networks with Primary User Recognition, in Cognitive Radio Oriented Wireless Networks and Communications, Aug. 2007.

[zhang07] Jingbin Zhang, Gang Zhou, Chengdu Huang, Sang H. Son and John A. Stankovic, TMMAC: An Energy Efficient Multi-Channel MAC Protocol for Ad Hoc Networks, in the ICC 2007 proceedings

[zhao05] Jun Zhao, Haito Zheng and Guang-Hua Yang, Distributed Coordination in Dynamic Spectrum Allocation Networks, in New Frontiers in Dynamic Spectrum Access Networks, 2005. 


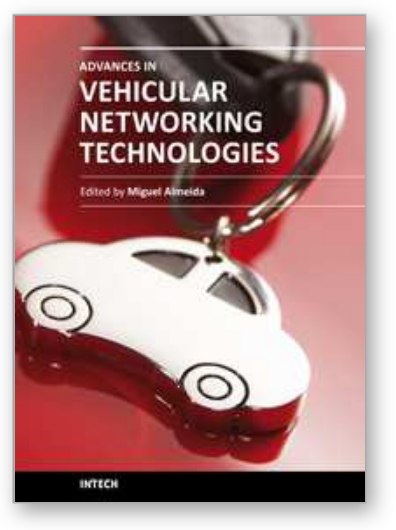

\author{
Advances in Vehicular Networking Technologies \\ Edited by Dr Miguel Almeida
}

ISBN 978-953-307-241-8

Hard cover, 432 pages

Publisher InTech

Published online 11, April, 2011

Published in print edition April, 2011

This book provides an insight on both the challenges and the technological solutions of several approaches, which allow connecting vehicles between each other and with the network. It underlines the trends on networking capabilities and their issues, further focusing on the MAC and Physical layer challenges. Ranging from the advances on radio access technologies to intelligent mechanisms deployed to enhance cooperative communications, cognitive radio and multiple antenna systems have been given particular highlight.

\title{
How to reference
}

In order to correctly reference this scholarly work, feel free to copy and paste the following:

Rodrigo Soulé de Castro, Philippe Godlewski and Philippe Martins (2011). An Overview of DSA via MultiChannel MAC Protocols, Advances in Vehicular Networking Technologies, Dr Miguel Almeida (Ed.), ISBN: 978953-307-241-8, InTech, Available from: http://www.intechopen.com/books/advances-in-vehicular-networkingtechnologies/an-overview-of-dsa-via-multi-channel-mac-protocols

\section{INTECH}

open science | open minds

\section{InTech Europe}

University Campus STeP Ri

Slavka Krautzeka 83/A

51000 Rijeka, Croatia

Phone: +385 (51) 770447

Fax: +385 (51) 686166

www.intechopen.com

\section{InTech China}

Unit 405, Office Block, Hotel Equatorial Shanghai

No.65, Yan An Road (West), Shanghai, 200040, China 中国上海市延安西路65号上海国际贵都大饭店办公楼 405 单元

Phone: +86-21-62489820

Fax: $+86-21-62489821$ 
(C) 2011 The Author(s). Licensee IntechOpen. This chapter is distributed under the terms of the Creative Commons Attribution-NonCommercialShareAlike-3.0 License, which permits use, distribution and reproduction for non-commercial purposes, provided the original is properly cited and derivative works building on this content are distributed under the same license. 